\title{
Scattering Amplitudes and the Conservative Hamiltonian for Binary Systems at Third Post-Minkowskian Order
}

\author{
Zvi Bern, ${ }^{1}$ Clifford Cheung, ${ }^{2}$ Radu Roiban, ${ }^{3}$ Chia-Hsien Shen, ${ }^{1}$ Mikhail P. Solon, ${ }^{2}$ and Mao Zeng ${ }^{4}$ \\ ${ }^{1}$ Mani L. Bhaumik Institute for Theoretical Physics, University of California at Los Angeles, Los Angeles, California 90095, USA \\ ${ }^{2}$ Walter Burke Institute for Theoretical Physics, California Institute of Technology, Pasadena, California 91125 \\ ${ }^{3}$ Institute for Gravitation and the Cosmos, Pennsylvania State University, University Park, Pennsylvania 16802, USA \\ ${ }^{4}$ Institute for Theoretical Physics, ETH Zürich, 8093 Zürich, Switzerland
}

(Received 28 January 2019; published 24 May 2019)

\begin{abstract}
We present the amplitude for classical scattering of gravitationally interacting massive scalars at third post-Minkowskian order. Our approach harnesses powerful tools from the modern amplitudes program such as generalized unitarity and the double-copy construction, which relates gravity integrands to simpler gauge-theory expressions. Adapting methods for integration and matching from effective field theory, we extract the conservative Hamiltonian for compact spinless binaries at third post-Minkowskian order. The resulting Hamiltonian is in complete agreement with corresponding terms in state-of-the-art expressions at fourth post-Newtonian order as well as the probe limit at all orders in velocity. We also derive the scattering angle at third post-Minkowskian order and find agreement with known results.
\end{abstract}

DOI: 10.1103/PhysRevLett.122.201603

Introduction.-The recent discovery of gravitational waves at LIGO/Virgo [1] has launched an extraordinary new era in astronomy, astrophysics and cosmology. Given expected improvements in detector sensitivity, high-precision theoretical predictions from general relativity will be crucial. Existing theory benchmarks come from a variety of approaches (see also Ref. [2] and references therein), including the effective one-body formalism [3], numerical relativity [4], the self-force formalism [5], and perturbative analysis using post-Newtonian (PN) [6-10], postMinkowskian (PM) [11-13], and effective field theory (EFT) [14] methods.

The past decade has also witnessed immense progress in the study of scattering amplitudes, where understanding mathematical structures within gauge theory and gravity has yielded new physical insights and efficient methods for calculation. In particular, the Bern-Carrasco-Johansson (BCJ) color-kinematics duality and associated double copy construction [15] allow multiloop gravitational amplitudes to be constructed from sums of products of gauge-theory quantities. This has yielded a variety of new results in supergravity (see Ref. [16] for recent results). The BCJ construction is intimately tied to the Kawai-Lewellen-Tye (KLT) relations [17], which relate tree amplitudes of closed and open strings.

Published by the American Physical Society under the terms of the Creative Commons Attribution 4.0 International license. Further distribution of this work must maintain attribution to the author(s) and the published article's title, journal citation, and DOI. Funded by SCOAP .
In this Letter, we apply modern amplitude methods to derive the classical scattering amplitude for two massive spinless particles at $\mathcal{O}\left(G^{3}\right)$ and to all orders in the velocity, i.e., at the third post-Minkowskian (3PM) order. We use generalized unitarity [18] to construct the corresponding two-loop integrand from tree amplitudes of gravitons and massive scalars, obtained straightforwardly from the double-copy construction. While the double copy introduces dilaton and antisymmetric tensor degrees of freedom [19], which are absent in pure Einstein gravity, we remove these unwanted states efficiently by restricting the state sums in unitarity cuts to gravitons alone. As we will show, we can calculate in strictly $D=4$ dimensions for the classical dynamics, where spinor helicity variables $[20,21]$ dramatically simplify the required tree amplitudes. The viability of working in $D=4$ offers optimism for extending our results to higher orders.

Afterwards, we integrate the two-loop integrand via a procedure adapted from EFT, in which energy integrals are evaluated in the potential region via residues before performing spatial integrations [22]. Using EFT matching $[22,23]$ we then derive the 3PM conservative Hamiltonian for compact spinless binaries. We show that the 4PN terms in our Hamiltonian are, up to a coordinate transformation, physically equivalent to corresponding terms in state-ofthe-art results. We also verify that our result agrees in the probe limit with the Hamiltonian for a test body orbiting a Schwarzschild black hole to 3PM order. Finally, we derive a compact expression for the 3PM scattering angle in terms of amplitude data.

Double copy and unitarity.-Dynamics at 3PM order is encoded in the two-loop scattering amplitude for two 

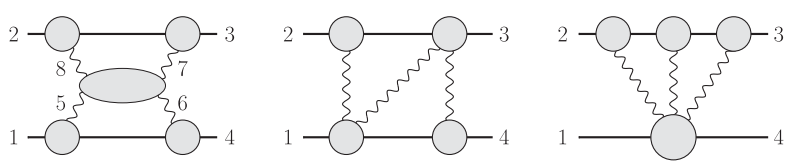

FIG. 1. Unitarity cuts needed for the classical scattering amplitude. The shaded ovals represent tree amplitudes while the exposed lines depict on-shell states. The wiggly and straight lines denote gravitons and massive scalars, respectively.

massive, gravitationally interacting scalars. Our calculation begins with a construction of the corresponding two-loop integrand via generalized unitarity. Because we are interested in classical scattering, we need not assemble the full quantum-mechanical integrand. Rather, as emphasized in Refs. [22-24], the classical potential only receives contributions with a single on-shell matter line per loop and with no gravitons starting and ending on the same matter line. For this reason we focus solely on the unitarity cuts shown in Fig. 1.

We obtain the tree amplitudes in the unitarity cuts via two methods. In the first approach, we work in general $D$ space-time dimensions. Exploiting color-kinematics duality [15], we derive gravitational amplitudes straightforwardly from simpler gauge-theory amplitudes by replacing color factors with corresponding kinematic factors. For the unitarity cuts of the classical limit of the two-loop scattering amplitude, the reference momenta that complicate projection onto graviton physical states can be eliminated, simplifying the calculation [25]. The primary purpose of our $D$-dimensional construction is to confirm explicitly the completeness of our second method, where we work in strictly $D=4$ so as to benefit from very simple expressions for gauge-theory amplitudes in terms of spinor helicity [20] variables. We then build the two corresponding gravitational amplitudes via the KLT relations [17]. At two loops, both approaches are efficient, but at higher loops, helicity amplitudes offer a much more compact starting point.

For concreteness, consider the first generalized unitarity cut in Fig. 1, which we refer to as $C^{\mathrm{H}-\mathrm{cut}}$ and is comprised of products of four 3-point and one 4-point amplitudes. Since four-point tree amplitudes are already very simple there is little computational advantage to imposing the on-shell conditions on matter lines. Thus, we replace the pairs of three-point amplitudes at the top and bottom of the cut with four-point amplitudes and then impose the matter cut conditions at the end. The resulting iterated two-particle cut is then

$$
\begin{aligned}
C^{2,2}= & \sum_{\text {states }} M_{4}\left(2^{s},-8,7,3^{s}\right) M_{4}(-5,6,-7,8) \\
& \times M_{4}\left(1^{s}, 5,-6,4^{s}\right),
\end{aligned}
$$

where $M_{4}$ denotes the tree-level four-point amplitude for gravity minimally coupled to two massive scalars denoted here by legs $1^{s}, 2^{s}, 3^{s}$, and $4^{s}$. In this cut, legs $1^{s}, 4^{s}$ have mass $m_{1}$ while legs $2^{s}, 3^{s}$ have mass $m_{2}$. All momenta in each tree amplitude are taken to be outgoing. The sum runs over graviton states for legs 5, 6, 7, and 8, where the minus signs on the labels indicate reversed momenta.

The four-point gravity tree amplitudes needed in the cuts are obtained from gauge-theory ones via the field-theory limit of KLT relations [17],

$$
M_{4}(1,2,3,4)=-i s_{12} A_{4}(1,2,3,4) A_{4}(1,2,4,3),
$$

where the $A_{4}$ are tree-level color-ordered gauge-theory four-point amplitudes and $s_{i j}=\left(p_{i}+p_{j}\right)^{2}$, working in mostly minus metric signature throughout. Strictly speaking, the KLT relations apply only to massless states. However, they can be applied here by interpreting the scalar masses, in the sense of dimensional reduction, as extradimensional momentum components. While we have not included coupling constants, these are easily restored at the end of the calculation by including an overall factor of $(8 \pi G)^{3}$, where $G$ is Newton's constant.

In terms of the spinor-helicity conventions of Ref. [21], the independent tree-level gauge-theory amplitudes needed in Eq. (1) are

$$
\begin{aligned}
A_{4}\left(1^{s}, 2^{+}, 3^{+}, 4^{s}\right) & =i \frac{m_{1}^{2}[23]}{\langle 23\rangle t_{12}}, \\
A_{4}\left(1^{s}, 2^{+}, 3^{-}, 4^{s}\right) & =i \frac{\langle 3|1| 2]^{2}}{t_{23} t_{12}}, \\
A_{4}\left(1^{-}, 2^{-}, 3^{+}, 4^{+}\right) & =i \frac{\langle 12\rangle^{4}}{\langle 12\rangle\langle 23\rangle\langle 34\rangle\langle 41\rangle}, \\
A_{4}\left(1^{-}, 2^{+}, 3^{-}, 4^{+}\right) & =i \frac{\langle 13\rangle^{4}}{\langle 12\rangle\langle 23\rangle\langle 34\rangle\langle 41\rangle},
\end{aligned}
$$

where $t_{i j}=2 p_{i} p_{j}$ and the \pm denote gluon helicities.

The dilaton and antisymmetric tensor states are removed from unitarity cuts by correlating the gluon helicities on both sides of the double copy. The unwanted states correspond to one gluon in the double copy of positive helicity and the other of negative helicity. An internal graviton state is obtained by taking the corresponding gluons in the KLT formula in Eq. (2) to be of the same helicity.

Using spinor evaluation techniques, it is straightforward to obtain a compact expression for the iterated two-particle cut in Eq. (1) (e.g., see Ref. [26]). Imposing cuts on the matter lines, as indicated in the first unitarity cut of Fig. 1, further simplifies it and gives $C^{\mathrm{H} \text {-cut }}$. We find

$$
\begin{aligned}
C^{\mathrm{H}-\mathrm{cut}}= & 2 i\left(\frac{1}{\left(p_{5}-p_{8}\right)^{2}}+\frac{1}{\left(p_{5}+p_{7}\right)^{2}}\right) \\
& \times\left(s_{23}^{2} m_{1}^{4} m_{2}^{4}+\frac{1}{s_{23}^{6}} \sum_{i=1,2}\left(\mathcal{E}_{i}^{4}+\mathcal{O}_{i}^{4}+6 \mathcal{O}_{i}^{2} \mathcal{E}_{i}^{2}\right)\right),
\end{aligned}
$$


where we have defined

$$
\begin{aligned}
\mathcal{E}_{1}^{2} & =\frac{1}{4} s_{23}^{2}\left(t_{18} t_{25}-t_{12} t_{58}\right)^{2}, \quad \mathcal{O}_{1}^{2}=\mathcal{E}_{1}^{2}-m_{1}^{2} m_{2}^{2} s_{23}^{2} t_{58}^{2} \\
\mathcal{E}_{2}^{2} & =\frac{1}{4} s_{23}^{2}\left[t_{17} t_{25}-t_{12} t_{57}-s_{23}\left(t_{17}+t_{57}\right)\right]^{2} \\
\mathcal{O}_{2}^{2} & =\mathcal{E}_{2}^{2}-m_{1}^{2} m_{2}^{2} s_{23}^{2} t_{57}^{2}
\end{aligned}
$$

The simplicity of this expression is a reflection of the double-copy structure: the same building blocks appear in the simpler corresponding gauge-theory cut.

The spurious double pole in $s_{23}$ can be explicitly cancelled by adding terms proportional to the Gram determinant formed from the five independent momenta at two loops that vanishes in $D=4$. In fact, the expression derived from the $D$-dimensional approach is automatically free of such spurious singularities. While these Gram determinants contribute quantum mechanically, we have checked explicitly that they vanish in the classical limit. This is not accidental-such terms are of the wrong form to generate the required $\log \left(s_{23}\right)$ needed to contribute to the classical 3PM amplitude (see Ref. [25] for details).

The remaining two independent generalized unitarity cuts in Fig. 2 are more complicated because they require five-point tree amplitudes with two massive scalar legs. The four-dimensional input gauge-theory amplitudes are simple to compute using modern methods (e.g., see Ref. [27]). For our $D$ construction we obtain a BCJ representation, allowing us to express the gravity cuts directly in terms of local diagrams. The particular representation was chosen such that we can ignore the reference momenta when projecting the internal states into gravitons. Further details will be given elsewhere [25].

To facilitate integration, we merge the cuts into a single integrand whose cuts match those in Fig. 1. This is achieved using an ansatz in terms of eight independent diagrams with only cubic vertices displayed in Fig. 2. The diagrammatic numerators are polynomials of the appropriate dimension exhibiting the symmetries of the corresponding diagram. Their coefficients are then fixed via the method of maximal cuts [28], whereby cuts of the integrand are constrained to match the known ones. This approach is sufficient for the two-loop problem.
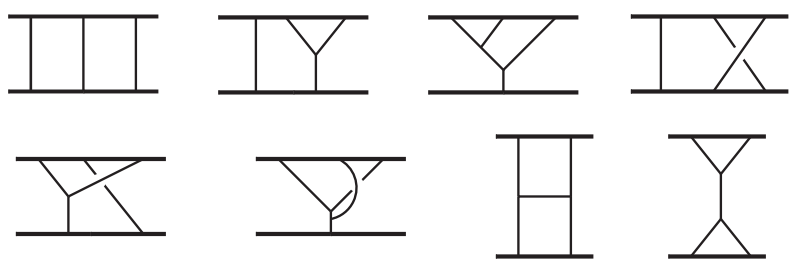

FIG. 2. The eight independent diagrams showing the propagator structure of integrals from which the classical contributions are extracted.
Integration.-Our method of integration follows Ref. [22]. For convenience, we give a short summary here, leaving details to Ref. [25]. Terms in the integrand take the form,

$$
\mathcal{I}=\frac{\text { numerator }}{\text { graviton propagators }} \times \prod_{i} \frac{1}{\omega_{i}^{2}-\boldsymbol{k}_{i}^{2}-m_{i}^{2}},
$$

where $i$ labels each matter line, which has energy $\omega_{i}$, spatial momentum $\boldsymbol{k}_{i}$, and mass $m_{i}$. The matter propagators can be factored into particle and antiparticle poles, $\omega_{i} \pm \sqrt{\boldsymbol{k}_{i}^{2}+m_{i}^{2}}$. We then express the integrand as $\mathcal{I}=\mathcal{N} \times \prod_{i}\left(1 / z_{i}\right)$, i.e., in terms of the particle poles $z_{i}=\omega_{i}-\sqrt{\boldsymbol{k}_{i}^{2}+m_{i}^{2}}$ and an effective numerator $\mathcal{N}$ that absorbs the rest of the integrand.

Following the procedure outlined in Ref. [22], we first evaluate the energy integrals. At two loops, i.e., 3PM order, we integrate over two independent combinations of energies, $\omega$ and $\omega^{\prime}$, in the potential region. As we will prove in detail in Ref. [25], the result is

$$
\tilde{\mathcal{I}}=\int \frac{d \omega}{2 \pi} \frac{d \omega^{\prime}}{2 \pi} \mathcal{I}\left(\omega, \omega^{\prime}\right)=\sum_{(i, j)} S_{i j} \operatorname{Res} \mathcal{I}\left(\omega, \omega^{\prime}\right),
$$

where the sum runs over distinct pairings $(i, j)$ of matter poles and $z_{i}=z_{j}=0$ when $\left(\omega, \omega^{\prime}\right)=\left(\omega_{i j}, \omega_{i j}^{\prime}\right)$. Here $S_{i j}$ is a calculable symmetry factor whose sign and magnitude depend on the topology of the cut graph. Note that the residue for an $(i, j)$ pairing will vanish if there are no values of $\omega$ and $\omega^{\prime}$ for which $z_{i}=z_{j}=0$.

The resulting quantity $\tilde{\mathcal{I}}$ depends on two independent spatial loop momenta. To integrate over them we employ dimensional regularization to deal with ultraviolet divergences stemming from the renormalization of delta function contact interactions, which do not contribute classically. Due to the localization on energy residues, $\tilde{\mathcal{I}}$ is a complicated, nonpolynomial function of three-dimensional invariants involving square roots. Nevertheless, we can series expand $\tilde{\mathcal{I}}$ in large $m_{1,2}$, yielding polynomials of kinematic invariants, which we can integrate at each order. After expanding, nearly all the spatial integrals are simple bubbles for which there are known analytic expressions [29]. The remaining integrals are evaluated via integration-by-parts identities [30].

For diagrams free from infrared (IR) singularities generated by iterations of lower-loop graviton exchanges, we have checked that our integrated results accord with several standard methods in the Feynman integral literature, including the Mellin-Barnes representation [29,31], numerical integration via sector decomposition [32], and differential equations [33] derived through integrationby-parts reduction [30,34]. The system of differential equations omits integrals lacking support on the matter pole residues that produce the classical contributions.

Amplitude and potential.-The integration procedure outlined above yields the conservative, i.e., real component 
of the 3PM amplitude generated by potential gravitons order by order in the large-mass expansion. Combining an explicit evaluation of this amplitude up to 7PN order with knowledge of the pole structure of individual integrals and exact, manifestly relativistic analytic results for certain graph topologies, we conjecture a full, all orders in velocity expression for the conservative 3PM amplitude (whose uniqueness will be discussed in Ref. [25]):

$$
\begin{aligned}
\mathcal{M}_{3}= & \frac{\pi G^{3} \nu^{2} m^{4} \log \boldsymbol{q}^{2}}{6 \gamma^{2} \xi}\left[3-6 \nu+206 \nu \sigma-54 \sigma^{2}+108 \nu \sigma^{2}\right. \\
& +4 \nu \sigma^{3}-\frac{48 \nu\left(3+12 \sigma^{2}-4 \sigma^{4}\right) \operatorname{arcsinh} \sqrt{\frac{\sigma-1}{2}}}{\sqrt{\sigma^{2}-1}} \\
& \left.-\frac{18 \nu \gamma\left(1-2 \sigma^{2}\right)\left(1-5 \sigma^{2}\right)}{(1+\gamma)(1+\sigma)}\right] \\
& +\frac{8 \pi^{3} G^{3} \nu^{4} m^{6}}{\gamma^{4} \xi}\left[3 \gamma\left(1-2 \sigma^{2}\right)\left(1-5 \sigma^{2}\right) F_{1}\right. \\
& \left.-32 m^{2} \nu^{2}\left(1-2 \sigma^{2}\right)^{3} F_{2}\right],
\end{aligned}
$$

where the log scale dependence is absorbed into a deltafunction ultraviolet counterterm. Here we use center-ofmass coordinates where the incoming and outgoing particle momenta are $\pm \boldsymbol{p}$ and $\pm(\boldsymbol{p}-\boldsymbol{q})$, respectively. We emphasize that $\mathcal{M}_{3}$ includes the nonrelativistic normalization factor, $1 / 4 E_{1} E_{2}$, where $E_{1,2}=\sqrt{\boldsymbol{p}^{2}+m_{1,2}^{2}}$. We also define the total mass $m=m_{1}+m_{2}$, the symmetric mass ratio $\nu=m_{1} m_{2} / m^{2}$, the total energy $E=E_{1}+E_{2}$, the symmetric energy ratio $\xi=E_{1} E_{2} / E^{2}$, the energy-mass ratio $\gamma=E / m$, and the relativistic kinematic invariant $\sigma=p_{1} \cdot p_{2} / m_{1} m_{2}$. We emphasize that Eq. (8) is not valid for $m_{1,2} \rightarrow 0$ since quantum terms of order $|\boldsymbol{q}| / m_{1,2}$ are dropped, as will be elaborated on in Ref. [25]. Also, note that the arcsinh factor is proportional to the sum of particle rapidities, $\operatorname{arctanh}|\boldsymbol{p}| / E_{1,2}$.

Equation (8) only includes $\boldsymbol{q}$-dependent terms that persist in the classical limit. The $\log \boldsymbol{q}^{2}$ term ultimately feeds into the conservative Hamiltonian through the Fourier transform $\left[\log \boldsymbol{q}^{2}\right]_{\mathrm{FT}}=-1 / 2 \pi|\boldsymbol{r}|^{3}$. The IR-divergent contributions, parametrized by $F_{1}=\int_{k_{1}} 1 / X_{1}^{2} Y_{1} X_{2}$ and $F_{2}=$ $\int_{\boldsymbol{k}_{1}, \boldsymbol{k}_{2}} 1 / X_{1}^{2} Y_{1} X_{2}^{2} Y_{2} X_{3}^{2}$ in the notation described in Eq. (12) of Ref. [22], will cancel in the EFT matching.

The Hamiltonian is extracted from the amplitude via EFT methods developed in Refs. [22,23,35] (see Ref. [13] for another approach). Consider massive spinless particles interacting via the center-of-mass Hamiltonian

$$
\begin{aligned}
& H(\boldsymbol{p}, \boldsymbol{r})=\sqrt{\boldsymbol{p}^{2}+m_{1}^{2}}+\sqrt{\boldsymbol{p}^{2}+m_{2}^{2}}+V(\boldsymbol{p}, \boldsymbol{r}), \\
& V(\boldsymbol{p}, \boldsymbol{r})=\sum_{i=1}^{\infty} c_{i}\left(\boldsymbol{p}^{2}\right)\left(\frac{G}{|\boldsymbol{r}|}\right)^{i},
\end{aligned}
$$

where $\boldsymbol{r}$ is the distance vector between particles and $i$ labels PM orders. The above Hamiltonian is in a gauge in which terms involving $\boldsymbol{p} \cdot \boldsymbol{r}$ or time derivatives of $\boldsymbol{p}$ are absent. We then compute the scattering amplitude of massive scalars, $\mathcal{M}^{(\mathrm{EFT})}=\sum_{i=1}^{\infty} \mathcal{M}_{i}^{(\mathrm{EFT})}$, where $\mathcal{M}_{3}^{(\mathrm{EFT})}$ comes from diagrams with two or fewer loops that depend on $c_{1}, c_{2}$, and $c_{3}$. In Ref. [22], the coefficients $c_{1}$ and $c_{2}$ were extracted analytically to all orders in velocity. Inserting these into $\mathcal{M}_{3}^{(\mathrm{EFT})}$ effectively implements the subtraction of iterated contributions. By equating $\mathcal{M}_{3}^{(\mathrm{EFT})}=\mathcal{M}_{3}$, we solve for the 3PM coefficient $c_{3}$.

The main result of the present Letter is the 3PM potential, encapsulated in the coefficients

$$
\begin{aligned}
c_{1}= & \frac{\nu^{2} m^{2}}{\gamma^{2} \xi}\left(1-2 \sigma^{2}\right), \\
c_{2}= & \frac{\nu^{2} m^{3}}{\gamma^{2} \xi}\left[\frac{3}{4}\left(1-5 \sigma^{2}\right)-\frac{4 \nu \sigma\left(1-2 \sigma^{2}\right)}{\gamma \xi}-\frac{\nu^{2}(1-\xi)\left(1-2 \sigma^{2}\right)^{2}}{2 \gamma^{3} \xi^{2}}\right], \\
c_{3}= & \frac{\nu^{2} m^{4}}{\gamma^{2} \xi}\left[\frac{1}{12}\left(3-6 \nu+206 \nu \sigma-54 \sigma^{2}+108 \nu \sigma^{2}+4 \nu \sigma^{3}\right)\right. \\
& -\frac{4 \nu\left(3+12 \sigma^{2}-4 \sigma^{4}\right) \operatorname{arcsinh} \sqrt{\frac{\sigma-1}{2}}}{\sqrt{\sigma^{2}-1}} \\
& -\frac{3 \nu \gamma\left(1-2 \sigma^{2}\right)\left(1-5 \sigma^{2}\right)}{2(1+\gamma)(1+\sigma)}-\frac{3 \nu \sigma\left(7-20 \sigma^{2}\right)}{2 \gamma \xi} \\
& -\frac{\nu^{2}\left(3+8 \gamma-3 \xi-15 \sigma^{2}-80 \gamma \sigma^{2}+15 \xi \sigma^{2}\right)\left(1-2 \sigma^{2}\right)}{4 \gamma^{3} \xi^{2}} \\
& \left.+\frac{2 \nu^{3}(3-4 \xi) \sigma\left(1-2 \sigma^{2}\right)^{2}}{\gamma^{4} \xi^{3}}+\frac{\nu^{4}(1-2 \xi)\left(1-2 \sigma^{2}\right)^{3}}{2 \gamma^{6} \xi^{4}}\right],
\end{aligned}
$$

where, for convenience, the expressions for $c_{1}$ and $c_{2}$ in Ref. [22] are reproduced here with slightly different normalization and in our current notation. As emphasized in Ref. [22], the cancellation of IR divergences between $\mathcal{M}_{3}^{(\mathrm{EFT})}$ and $\mathcal{M}_{3}$ depends critically on $c_{1}$ and $c_{2}$ and thus provides a nontrivial check of our calculation.

Consistency checks.-Our results pass several nontrivial albeit overlapping consistency checks (see Ref. [25] for details). First and foremost, we have verified that the 4PN terms in our Hamiltonian are equivalent to known results up to a canonical coordinate transformation,

$$
\begin{aligned}
& (\boldsymbol{r}, \boldsymbol{p}) \rightarrow(\boldsymbol{R}, \boldsymbol{P})=(A \boldsymbol{r}+B \boldsymbol{p}, C \boldsymbol{p}+D \boldsymbol{r}) \\
& A=1-\frac{G m \nu}{2|\boldsymbol{r}|}+\cdots, \quad B=\frac{G(1-2 / \nu)}{4 m|\boldsymbol{r}|} \boldsymbol{p} \cdot \boldsymbol{r}+\cdots, \\
& C=1+\frac{G m \nu}{2|\boldsymbol{r}|}+\cdots, \quad D=-\frac{G m \nu}{2|\boldsymbol{r}|^{3}} \boldsymbol{p} \cdot \boldsymbol{r}+\cdots,
\end{aligned}
$$

with ellipses denoting higher order corrections entering as a power series in $G /|\boldsymbol{r}|, \boldsymbol{p}^{2}$, and $(\boldsymbol{p} \cdot \boldsymbol{r})^{2} / \boldsymbol{r}^{2}$ (for past 
treatments, see Ref. [36,37]). To derive this coordinate transformation we generate an ansatz for $A, B, C$, and $D$ and constrain it to preserve the Poisson brackets, i.e., $\{\boldsymbol{r}, \boldsymbol{p}\}=\{\boldsymbol{R}, \boldsymbol{P}\}=\mathbf{1}$ with all other brackets vanishing, in the spirit of Ref. [38]. We verify that within this space of canonical transformations exists a subspace that maps our Hamiltonian in Eq. (10) to the one in the literature, e.g., as summarized in Eq. (8.41) of Ref. [10], up to the intersection of $3 \mathrm{PM}$ and 4PN accuracy.

Second, applying the methods of Ref. [22], we have checked that the full-theory amplitude $\mathcal{M}_{3}$ in Eq. (8) is identical to the amplitude $\mathcal{M}_{3}^{(\mathrm{EFT})}$ computed from the conservative Hamiltonian in Ref. [10] up to 4PN accuracy.

Third, we have extracted from our Hamiltonian the coordinate invariant energy of a circular orbit as a function of the period. Working at $2 \mathrm{PN}$ order-the highest order subsumed by 3PM which is relevant to a virialized system-we agree with known results [8].

Fourth, by solving the equations of motion derived from our Hamiltonian, we obtain the 3PM-accurate classical scattering angle in the center-of-mass frame and neglecting radiation,

$$
2 \pi \chi=\frac{d_{1}}{J}+\frac{d_{2}}{J^{2}}+\frac{1}{J^{3}}\left(-4 d_{3}+\frac{d_{1} d_{2}}{\pi^{2}}-\frac{d_{1}^{3}}{48 \pi^{2}}\right),
$$

where $J=b|\boldsymbol{p}|$ is the angular momentum, $b$ is the impact parameter, and we have defined $d_{1}=m \gamma \xi \boldsymbol{q}^{2} \mathcal{M}_{1}^{\prime} /|\mathbf{p}|$, $d_{2}=m \gamma \xi|\boldsymbol{q}| \mathcal{M}_{2}^{\prime}$, and $d_{3}=m \gamma \xi|\mathbf{p}| \mathcal{M}_{3}^{\prime} / \log \boldsymbol{q}^{2}$, where the $\boldsymbol{q}$ dependence cancels. The primed quantities denote the IRfinite parts of the nonrelativistically normalized amplitudes that enter the Hamiltonian coefficients as defined here and in Ref. [22], so

$$
\begin{aligned}
& \mathcal{M}_{1}^{\prime}=-\frac{4 \pi G \nu^{2} m^{2}}{\gamma^{2} \xi \boldsymbol{q}^{2}}\left(1-2 \sigma^{2}\right), \\
& \mathcal{M}_{2}^{\prime}=-\frac{3 \pi^{2} G^{2} \nu^{2} m^{3}}{2 \gamma^{2} \xi|\boldsymbol{q}|}\left(1-5 \sigma^{2}\right),
\end{aligned}
$$

and $\mathcal{M}_{3}^{\prime}$ is the $\log \boldsymbol{q}^{2}$ term in Eq. (8). Truncated to $4 \mathrm{PN}$ order, Eq. (12) agrees with known results [39].

Last but not least, in the probe limit $m_{1} \ll m_{2}$, our result exactly coincides with the Hamiltonian for a point particle in a Schwarzschild background to $\mathcal{O}\left(G^{3}\right)$ and all orders in velocity, e.g., as given in Eq. (8) of Ref. [40].

Conclusions. - We have presented the 3PM amplitude for classical scattering of gravitationally interacting massive spinless particles. From this amplitude we have extracted the corresponding conservative Hamiltonian for binary dynamics to $3 \mathrm{PM}$ order.

The 3PM Hamiltonian in Eqs. (9) and (10) will be employed in a forthcoming paper [41] to compute approximants for the binding energy of binary systems moving on circular orbits and assess their accuracy against numerical-relativity predictions. This is relevant for understanding the usefulness of PM calculations when building accurate waveform models for LIGO/Virgo data analysis.

Our Letter leaves many avenues for future work, e.g., including obtaining higher orders in the PM expansion, incorporating spin [42], radiation [43], and finite-size effects, as well as connecting to other recent amplitude approaches $[19,44]$ and the effective one-body formalism $[3,12,13,45]$.

The simplicity of the 3PM amplitude in Eq. (8) and potential in Eq. (10) bodes well for future progress. Moreover, since the amplitude and EFT methods employed in this Letter are far from exhausted, we believe that the results we have reported mark only the beginning.

We thank Alessandra Buonanno, Thibault Damour, Michael Enciso, David Kosower, Andrés Luna, Aneesh Manohar, Smadar Naoz, Julio Parra-Martinez, Rafael Porto, Jan Steinhoff, George Sterman, Justin Vines, and Mark Wise for helpful discussions, including comments on the manuscript. In addition, we especially thank Ira Rothstein for his many insightful comments throughout this project. Z. B. is supported by the U.S. Department of Energy (DOE) under Award No. DE-SC0009937. C. C. is supported by the DOE under Grant No. DE-SC0011632. R. R. is supported by the U.S. Department of Energy (DOE) under Grant No. DE-SC0013699. C. H. S. is supported by the Mani L. Bhaumik Institute for Theoretical Physics. M.P. S. is supported by the DOE under Grant No. DE-SC0011632 and the McCone Fellowship at the Walter Burke Institute. M.Z. is supported by the Swiss National Science Foundation under Contract No. SNF200021179016 and the European Commission through the ERC Grant pertQCD.

[1] B. P. Abbott et al. (LIGO Scientific and Virgo Collaborations), Observation of Gravitational Waves from a Binary Black Hole Merger, Phys. Rev. Lett. 116, 061102 (2016); Observation of Gravitational Waves from a Binary Neutron Star Inspiral, Phys. Rev. Lett. 119, 161101 (2017).

[2] L. Blanchet, Gravitational radiation from post-Newtonian sources and inspiralling compact binaries, Living Rev. Relativity 17, 2 (2014); R. A. Porto, The effective field theorists approach to gravitational dynamics, Phys. Rep. 633, 1 (2016); G. Schäfer and P. Jaranowski, Hamiltonian formulation of general relativity and post-Newtonian dynamics of compact binaries, Living Rev. Relativity 21, 7 (2018); L. Barack and A. Pound, Self-force and radiation reaction in general relativity, Rep. Prog. Phys. 82, 016904 (2019); M. Levi, Effective field theories of post-Newtonian gravity: A comprehensive review, arXiv:1807.01699.

[3] A. Buonanno and T. Damour, Effective one-body approach to general relativistic two-body dynamics, Phys. Rev. D 59, 084006 (1999); Transition from inspiral to plunge in binary black hole coalescences, Phys. Rev. D 62, 064015 (2000). 
[4] F. Pretorius, Evolution of Binary Black Hole Spacetimes, Phys. Rev. Lett. 95, 121101 (2005); M. Campanelli, C. O. Lousto, P. Marronetti, and Y. Zlochower, Accurate Evolutions of Orbiting Black-Hole Binaries without Excision, Phys. Rev. Lett. 96, 111101 (2006); J. G. Baker, J. Centrella, D. I. Choi, M. Koppitz, and J. van Meter, Gravitational Wave Extraction from an Inspiraling Configuration of Merging Black Holes, Phys. Rev. Lett. 96, 111102 (2006).

[5] Y. Mino, M. Sasaki, and T. Tanaka, Gravitational radiation reaction to a particle motion, Phys. Rev. D 55, 3457 (1997); T. C. Quinn and R. M. Wald, An axiomatic approach to electromagnetic and gravitational radiation reaction of particles in curved space-time, Phys. Rev. D 56, 3381 (1997).

[6] J. Droste, The field of $n$ moving centres in Einsteins theory of gravitation, Proc. Acad. Sci. Amsterdam 19, 197 (1916); A. Einstein, L. Infeld, and B. Hoffmann, The gravitational equations and the problem of motion, Ann. Math. (N.Y.) 39, 65 (1938); T. Ohta, H. Okamura, T. Kimura, and K. Hiida, Physically acceptable solution of Einstein's equation for many-body system, Prog. Theor. Phys. 50, 492 (1973).

[7] P. Jaranowski and G. Schäfer, Third post-Newtonian higher order ADM Hamilton dynamics for two-body point mass systems, Phys. Rev. D 57, 7274 (1998); Erratum, Phys. Rev. D 63, 029902(E) (2000).

[8] T. Damour, P. Jaranowski, and G. Schäfer, Dynamical invariants for general relativistic two-body systems at the third post-Newtonian approximation, Phys. Rev. D 62, 044024 (2000); L. Blanchet and G. Faye, Equations of motion of point particle binaries at the third post-Newtonian order, Phys. Lett. A 271, 58 (2000); T. Damour, P. Jaranowski, and G. Schäfer, Dimensional regularization of the gravitational interaction of point masses, Phys. Lett. B 513, 147 (2001).

[9] T. Damour, P. Jaranowski, and G. Schäfer, Nonlocal-intime action for the fourth post-Newtonian conservative dynamics of two-body systems, Phys. Rev. D 89, 064058 (2014).

[10] P. Jaranowski and G. Schäfer, Derivation of local-in-time fourth post-Newtonian ADM Hamiltonian for spinless compact binaries, Phys. Rev. D 92, 124043 (2015).

[11] B. Bertotti, On gravitational motion, Nuovo Cimento 4, 898 (1956); R. P. Kerr, The Lorentz-covariant approximation method in general relativity, I. Nuovo Cimento 13, 469 (1959); B. Bertotti and J. F. Plebański, Theory of gravitational perturbations in the fast motion approximation, Ann. Phys. (N.Y.) 11, 169 (1960); M. Portilla, Momentum and angular momentum of two gravitating particles, J. Phys. A 12, 1075 (1979); K. Westpfahl and M. Goller, Gravitational scattering of two relativistic particles in postlinear approximation, Lett. Nuovo Cimento 26, 573 (1979); M. Portilla, Scattering of two gravitating particles: Classical approach, J. Phys. A 13, 3677 (1980); L. Bel, T. Damour, N. Deruelle, J. Ibanez, and J. Martin, Poincaré-invariant gravitational field and equations of motion of two pointlike objects: The postlinear approximation of general relativity, Gen. Relativ. Gravit. 13, 963 (1981); K. Westpfahl, High speed scattering of charged and uncharged particles in general relativity, Fortschr. Phys. 33, 417 (1985).
[12] T. Damour, Gravitational scattering, post-Minkowskian approximation and effective one-body theory, Phys. Rev. D 94, 104015 (2016).

[13] T. Damour, High-energy gravitational scattering and the general relativistic two-body problem, Phys. Rev. D 97, 044038 (2018).

[14] W. D. Goldberger and I. Z. Rothstein, An effective field theory of gravity for extended objects, Phys. Rev. D 73, 104029 (2006).

[15] Z. Bern, J. J. M. Carrasco, and H. Johansson, New relations for gauge-theory amplitudes, Phys. Rev. D 78, 085011 (2008); Perturbative Quantum Gravity as a Double Copy of Gauge Theory, Phys. Rev. Lett. 105, 061602 (2010).

[16] Z. Bern, J. J. M. Carrasco, L. J. Dixon, H. Johansson, and R. Roiban, Simplifying multiloop integrands and ultraviolet divergences of gauge theory and gravity amplitudes, Phys. Rev. D 85, 105014 (2012); Z. Bern, J. J. Carrasco, W. M. Chen, A. Edison, H. Johansson, J. Parra-Martinez, R. Roiban, and M. Zeng, Ultraviolet properties of $\mathcal{N}=8$ supergravity at five loops, Phys. Rev. D 98, 086021 (2018).

[17] H. Kawai, D. C. Lewellen, and S. H. H. Tye, A relation between tree amplitudes of closed and open strings, Nucl. Phys. B269, 1 (1986).

[18] Z. Bern, L. J. Dixon, D. C. Dunbar, and D. A. Kosower, One loop $n$-point gauge theory amplitudes, unitarity and collinear limits, Nucl. Phys. B425, 217 (1994); Z. Bern, L. J. Dixon, D. C. Dunbar, and D. A. Kosower, Fusing gauge theory tree amplitudes into loop amplitudes, Nucl. Phys. B435, 59 (1995).

[19] H. Johansson and A. Ochirov, Pure gravities via colorkinematics duality for fundamental matter, J. High Energy Phys. 11 (2015) 046; A. Luna, I. Nicholson, D. O'Connell, and C.D. White, Inelastic black hole scattering from charged scalar amplitudes, J. High Energy Phys. 03 (2018) 044.

[20] F. A. Berends, R. Kleiss, P. De Causmaecker, R. Gastmans, and T. T. Wu, Single bremsstrahlung processes in gauge theories, Phys. Lett. 103B, 124 (1981); F. A. Berends, R. Kleiss, P. De Causmaecker, R. Gastmans, W. Troost, and T. T. Wu, Multiple bremsstrahlung in gauge theories at highenergies. 2. Single bremsstrahlung, Nucl. Phys. B206, 61 (1982); Z. Xu, D. H. Zhang, and L. Chang, Helicity amplitudes for multiple bremsstrahlung in massless nonabelian gauge theories, Nucl. Phys. B291, 392 (1987).

[21] M. L. Mangano and S. J. Parke, Multiparton amplitudes in gauge theories, Phys. Rep. 200, 301 (1991).

[22] C. Cheung, I. Z. Rothstein, and M. P. Solon, From Scattering Amplitudes to Classical Potentials in the Post-Minkowskian Expansion, Phys. Rev. Lett. 121, 251101 (2018).

[23] D. Neill and I. Z. Rothstein, Classical space-times from the S matrix, Nucl. Phys. B877, 177 (2013).

[24] N. E. J. Bjerrum-Bohr, P. H. Damgaard, G. Festuccia, L. Plante, and P. Vanhove, General Relativity from Scattering Amplitudes, Phys. Rev. Lett. 121, 171601 (2018).

[25] Z. Bern, C. Cheung, R. Roiban, C.-H. Shen, M. P. Solon, and M. Zeng (to be published).

[26] Z. Bern, L. J. Dixon, D. C. Dunbar, M. Perelstein, and J. S. Rozowsky, On the relationship between Yang-Mills theory and gravity and its implication for ultraviolet divergences, Nucl. Phys. B530, 401 (1998). 
[27] S. D. Badger, E. W. N. Glover, V. V. Khoze, and P. Svrcek, Recursion relations for gauge theory amplitudes with massive particles, J. High Energy Phys. 07 (2005) 025.

[28] Z. Bern, J. J. M. Carrasco, H. Johansson, and D. A. Kosower, Maximally supersymmetric planar Yang-Mills amplitudes at five loops, Phys. Rev. D 76, 125020 (2007).

[29] V. A. Smirnov, Analytic tools for Feynman integrals, Springer Tracts Mod. Phys. 250, 1 (2012).

[30] K. G. Chetyrkin and F. V. Tkachov, Integration by parts: The algorithm to calculate beta functions in 4 Loops, Nucl. Phys. B192, 159 (1981).

[31] V. A. Smirnov, Analytical result for dimensionally regularized massless on shell double box, Phys. Lett. B 460, 397 (1999); M. Czakon, Automatized analytic continuation of Mellin-Barnes integrals, Comput. Phys. Commun. 175, 559 (2006); A. V. Smirnov and V. A. Smirnov, On the resolution of singularities of multiple Mellin-Barnes integrals, Eur. Phys. J. C 62, 445 (2009); J. Gluza, K. Kajda, T. Riemann, and V. Yundin, Numerical evaluation of tensor Feynman integrals in Euclidean kinematics, Eur. Phys. J. C 71, 1516 (2011).

[32] T. Binoth and G. Heinrich, Numerical evaluation of multiloop integrals by sector decomposition, Nucl. Phys. B680, 375 (2004); A. V. Smirnov, FIESTA4: Optimized Feynman integral calculations with GPU support, Comput. Phys. Commun. 204, 189 (2016).

[33] A. V. Kotikov, Differential equations method: New technique for massive Feynman diagrams calculation, Phys. Lett. B 254, 158 (1991); Z. Bern, L. J. Dixon, and D. A. Kosower, Dimensionally regulated pentagon integrals, Nucl. Phys. B412, 751 (1994); E. Remiddi, Differential equations for Feynman graph amplitudes, Nuovo Cimento A 110, 1435 (1997); T. Gehrmann and E. Remiddi, Differential equations for two loop four point functions, Nucl. Phys. B580, 485 (2000).

[34] S. Laporta, High precision calculation of multiloop Feynman integrals by difference equations, Int. J. Mod. Phys. A 15, 5087 (2000); A. V. Smirnov, FIRE5: A C++ implementation of Feynman Integral REduction, Comput. Phys. Commun. 189, 182 (2015); P. Maierhöfer, J. Usovitsch, and P. Uwer, Kira-A Feynman integral reduction program, Comput. Phys. Commun. 230, 99 (2018).

[35] C. Cheung and M. P. Solon (to be published).

[36] N. E. J. Bjerrum-Bohr, J. F. Donoghue, and B. R. Holstein, Quantum gravitational corrections to the nonrelativistic scattering potential of two masses, Phys. Rev. D 67, 084033 (2003); Erratum, Phys. Rev. D 71, 069903(E) (2005).

[37] B. R. Holstein and A. Ross, Spin effects in long range gravitational scattering, arXiv:0802.0716.

[38] T. Damour, P. Jaranowski, and G. Schäfer, Poincare invariance in the ADM Hamiltonian approach to the general relativistic two-body problem, Phys. Rev. D 62, 021501 (2000); Erratum 63, 029903(E) (2000).

[39] D. Bini and T. Damour, Gravitational scattering of two black holes at the fourth post-Newtonian approximation, Phys. Rev. D 96, 064021 (2017).
[40] N. Wex and G. Schäfer, Innermost stable orbits for coalescing binary systems of compact objects-a remark, Classical Quantum Gravity 10, 2729 (1993).

[41] A. Antonelli, A. Buonanno, J Steinhoff, M. van de Meent, and J. Vines, Energetics of two-body Hamiltonians in postMinkowskian theory, arXiv:1901.07102.

[42] V. Vaidya, Gravitational spin Hamiltonians from the $\mathrm{S}$ matrix, Phys. Rev. D 91, 024017 (2015); A. Guevara, Holomorphic classical limit for spin effects in gravitational and electromagnetic scattering, arXiv:1706.02314; A. Guevara, A. Ochirov, and J. Vines, Scattering of spinning black holes from exponentiated soft factors, arXiv: 1812.06895; M. Z. Chung, Y. T. Huang, J. W. Kim, and $\mathrm{S}$. Lee, The simplest massive S-matrix: From minimal coupling to black holes, arXiv:1812.08752.

[43] A. Luna, R. Monteiro, I. Nicholson, D. O'Connell, and C. D. White, The double copy: Bremsstrahlung and accelerating black holes, J. High Energy Phys. 06 (2016) 023; W. D. Goldberger and A. K. Ridgway, Radiation and the classical double copy for color charges, Phys. Rev. D 95, 125010 (2017); W. D. Goldberger, S. G. Prabhu, and J. O. Thompson, Classical gluon and graviton radiation from the bi-adjoint scalar double copy, Phys. Rev. D 96, 065009 (2017); W. D. Goldberger and A. K. Ridgway, Bound states and the classical double copy, Phys. Rev. D 97, 085019 (2018); D. Chester, Radiative double copy for EinsteinYang-Mills theory, Phys. Rev. D 97, 084025 (2018); W. D. Goldberger, J. Li, and S. G. Prabhu, Spinning particles, axion radiation, and the classical double copy, Phys. Rev. D 97, 105018 (2018); J. Li and S. G. Prabhu, Gravitational radiation from the classical spinning double copy, Phys. Rev. D 97, 105019 (2018); C. H. Shen, Gravitational radiation from color-kinematics duality, J. High Energy Phys. 11 (2018) 162.

[44] F. Cachazo and A. Guevara, Leading singularities and classical gravitational scattering, arXiv:1705.10262; A. Laddha and A. Sen, Observational signature of the logarithmic terms in the soft graviton theorem, arXiv: 1806.01872; J. Plefka, J. Steinhoff, and W. Wormsbecher, Effective action of dilaton gravity as the classical double copy of Yang-Mills theory, Phys. Rev. D 99, 024021 (2019); B. Sahoo and A. Sen, Classical and quantum results on logarithmic terms in the soft theorem in four dimensions, J. High Energy Phys. 02 (2019) 086; D. A. Kosower, B. Maybee, and D. O'Connell, Amplitudes, observables, and classical scattering, J. High Energy Phys. 02 (2019) 137; M. Ciafaloni, D. Colferai, and G. Veneziano, Infrared features of gravitational scattering and radiation in the eikonal approach, arXiv:1812.08137.

[45] T. Damour, F. Guercilena, I. Hinder, S. Hopper, A. Nagar, and L. Rezzolla, Strong-field scattering of two black holes: Numerics versus analytics, Phys. Rev. D 89, 081503 (2014); J. Vines, Scattering of two spinning black holes in postMinkowskian gravity, to all orders in spin, and effectiveone-body mappings, Classical Quantum Gravity 35, 084002 (2018). 